\title{
Enhancement of Localization in One-Dimensional Random Potentials with Long-Range Correlations
}

\author{
U. Kuhl, ${ }^{1}$ F. M. Izrailev, ${ }^{2}$ and A. A. Krokhin ${ }^{2,3}$ \\ ${ }^{1}$ Fachbereich Physik, Philipps-Universität Marburg, Renthof 5, D-35032 Marburg, Germany \\ ${ }^{2}$ Instituto de Física, Universidad Autónoma de Puebla, Apartado Postal J-48, Puebla, Puebla, 72570, México \\ ${ }^{3}$ Department of Physics, University of North Texas, P.O. Box 311427, Denton, Texas 76203, USA \\ (Received 7 September 2007; revised manuscript received 9 November 2007; published 28 March 2008)
}

\begin{abstract}
We experimentally study the effect of enhancement of localization in weak one-dimensional random potentials. Our experimental setup is a single-mode waveguide with 100 tunable scatterers periodically inserted into the waveguide. By measuring the amplitudes of transmitted and reflected waves in the spacing between each pair of scatterers, we observe a strong decrease of the localization length when white-noise scatterers are replaced by a correlated arrangement of scatterers.
\end{abstract}

DOI: 10.1103/PhysRevLett.100.126402

PACS numbers: 71.23.An, 03.65.Nk, 42.25.Bs

Processes of destructive interference at backscattering do not vanish after averaging over disorder, unlike interference at scattering over other directions. In a random potential this property of backscattering may lead to Anderson localization [1]. Experimentally it is difficult to find Anderson localization for electrons due to Coulomb and electron-phonon interaction, but it was found for photons in optically diffusive media [2-4] (for a review see Ref. [5]). In case of white-noise one-dimensional potentials $U(x)$, where backscattering leads to localization at any energy $E$ independently of the strength of the potential, the localization length $l(E)$ is determined by spectral composition of the correlation function [6,7],

$$
\lambda(E) \equiv l^{-1}(E)=\left(\sigma^{2} / 8 k^{2}\right) W(2 k) .
$$

Here $k^{2}=E$ is the energy of an eigenstate, $W(2 k)$ is the Fourier harmonic of the correlator $\left\langle U(x) U\left(x^{\prime}\right)\right\rangle$, and $\sigma^{2}=$ $\left\langle U^{2}(x)\right\rangle-\langle U(x)\rangle^{2}$ is the variance of disorder. Equation (1) is obtained in the first (Born) approximation over weak disorder, i.e., $\sigma^{2} \ll 1$.

A common opinion is that the shortest localization length is reached for the most disordered (uncorrelated) potentials with white-noise spectrum $W(k)=1$. This opinion is based on the fact that correlations, reducing the degree of disorder, typically give rise to extended states. In the dimer model, short-range correlations result in two resonant extended states $[8,9]$, that was observed in a semiconductor superlattice [10]. A continuum of extended states for potentials with long-range correlations was predicted in Refs. [11,12] and was experimentally verified in a microwave waveguide with intentionally introduced correlated disorder [13]. Thus, there is a strong evidence that correlations may destroy localization [14].

In this Letter we address the opposite situation and show that correlations in weakly disordered potentials can enhance localization for a continuum of states, resulting in the localization length much shorter than that in a whitenoise potential with the same $\sigma$. The effect of localization enhancement is important for random lasers [15,16], where extremely localized states provide higher efficiency. Our results clearly demonstrate that the long-range correlations may either suppress or enhance localization. This conclusion probably has to change a common point of view that correlations, being a manifestation of some kind of order, may only suppress localization.

The setup is a single-mode waveguide with transverse dimensions $a=20 \mathrm{~mm}, b=10 \mathrm{~mm}$, and with 100 cylindrical scatterers of radius $r=2.5 \mathrm{~mm}$, periodically inserted with spacing of $d=20.5 \mathrm{~mm}$, see Fig. 1 . In the experiment, we have exploited the transmission and reflection of the lowest waveguide mode in the frequency range between $\nu_{\min }=c / 2 a \approx 7.5 \mathrm{GHz}$ and $\nu_{\max }=c / a=$ $c / 2 b \approx 15 \mathrm{GHz}$. The dispersion relation in an empty rectangular microwave waveguide is given by $k=(2 \pi / c) \times$ $\sqrt{\nu^{2}-\nu_{\min }^{2}}$, where $c$ is the speed of light. Thus the normalized wave vector $k d / \pi$ ranges from 0 to 1.8. The setup was already used to show the "Hofstadter butterfly" [17] and mobility edges emerging from correlated disorder [13]. The arrangement allows to assemble a random potential with prescribed correlation function.

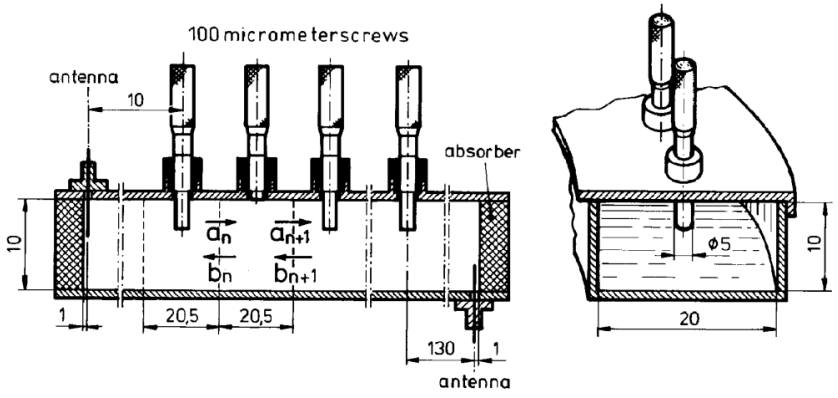

FIG. 1. Schematic view of the waveguide with 100 scatterers. The waveguide is closed by microwave absorbers at both ends. The lower antenna can be placed anywhere within the scattering arrangement. 
The strength $U_{n}$ of a single scatterer is associated with its length embedded inside the waveguide, and the length is varied by micrometer screws. When $U_{n}=$ const, the waveguide is fully transparent in the Bloch band. For the whitenoise potential 100 numbers $\varepsilon_{n}=U_{n}-\left\langle U_{n}\right\rangle$ were drawn as uncorrelated random numbers with variance $\sigma^{2}$. The corresponding localization length was much larger than the length $L$ of the waveguide; thus, the eigenstates are delocalized within the waveguide. However, if the white-noise potential $\varepsilon_{n}$ is replaced by a correlated sequence, the localization length inside a prescribed window of energy is strongly reduced and the effects of localization in the transmission and reflection become observable.

The experiment can be well described by a onedimensional equation of Kronig-Penney type [13],

$$
\psi^{\prime \prime}(x)+k^{2} \psi(x)=\sum_{n=-\infty}^{\infty} U_{n} \psi\left(x_{n}\right) \delta\left(x-x_{n}\right)
$$

Here $U_{n}$ is the amplitude of the $n$th delta scatterer located at $x=x_{n}=n d$. Therefore Eq. (1), which assumes an electron with parabolic dispersion, is replaced for an electromagnetic waveguide by [13]

$$
l^{-1}(E)=\frac{\sigma^{2} k^{2}}{8} \frac{\sin ^{2}(k d)}{\sin ^{2} \mu} W(2 \mu) .
$$

Here $\sigma^{2}=\left\langle U_{n}^{2}\right\rangle-\left\langle U_{n}\right\rangle^{2}$. The phase $\mu$ is given by the Kronig-Penney dispersion relation,

$$
2 \cos \mu=2 \cos (k d)+\frac{\left\langle U_{n}\right\rangle}{k} \sin (k d), \quad 0 \leq \mu \leq \pi .
$$

The function $W(2 \mu)$ is the Fourier transform of the binary correlator $\zeta(s)=\left\langle\varepsilon_{n} \varepsilon_{n+s}\right\rangle / \sigma^{2}$,

$$
W(2 \mu)=1+2 \sum_{s=1}^{\infty} \zeta(s) \cos (2 s \mu)
$$

It follows from Eq. (5) that $W(2 \mu)$ is symmetric with respect to the band center $\mu=\pi / 2$.

For any white-noise potential the correlator $\zeta(s) \equiv 0$, apart from $\zeta(0) \equiv 1$, leading to $W(2 \mu)=1$. To observe the effect of enhancement of localization it is necessary to have the function $W(2 \mu)=W_{0} \gg 1$ within some interval $\Delta \mu=\mu_{2}-\mu_{1}$. Because of the normalization condition

$$
\zeta(0)=\frac{2}{\pi} \int_{0}^{\pi / 2} W(2 \mu) d \mu=1,
$$

the width $\Delta \mu$ and the enhancement factor $W_{0}$ are related, $2 W_{0}=\pi \Delta \mu$, providing that $W(2 \mu)$ vanishes outside the interval $\left[\mu_{1}, \mu_{2}\right]$. Thus, the correlation-induced enhancement of localization within the interval $\Delta \mu$ is accompanied by full transparency of the waveguide for all other frequencies [18]. The Fourier coefficients of the step function, that is $W(2 \mu)=W_{0}$ within the interval $\Delta \mu$ and $W(2 \mu)=0$ otherwise, is given by

$$
\zeta(s)=\frac{1}{2 s} \frac{\sin \left(2 s \mu_{2}\right)-\sin \left(2 s \mu_{1}\right)}{\mu_{2}-\mu_{1}} .
$$

The inverse-power-law decay of $\zeta(s)$ is a signature of the long-range correlations. A correlated sequence was generated using the algorithm proposed in Ref. [13]. The two random sets used in the experiments are shown in Fig. 2, including their correlations.

For both sets the elements $S_{12}=S_{21}^{*}$ and $S_{22}$ of the scattering matrix were measured as functions of antenna position. Here $S_{12}\left(S_{22}\right)$ is the transmission (reflection) amplitude of the scattering process when the fixed antenna is in front of the first scatterer $(n=1)$ and the moving antenna is located between the $n$th and $(n+1)$ st scatterer. The moving antenna emits and receives the signal while measuring $S_{22}$.

The single-mode transmission patterns $\left|S_{12}\right|$ are shown in Fig. 3 for the purely random (upper) and correlated (lower) sequence (see also Fig. 2) as a function of antenna position (vertical axis) and wave number $k$ (horizontal axis). In addition, on top of each figure, we present the dependence of transmission value $\left|S_{12}\right|$ through the whole waveguide. As one can see, for the uncorrelated disorder there is a gap close to the edge of the Brillouin zone $k d / \pi=1$. It originates from the periodic spacing between the scatterers. For small wave numbers the transmission is small because of the weak antenna coupling to the waveguide, whereas for high wave numbers it is small due to large absorption. In case of the correlated disorder there are additional gaps located at $k d / \pi \approx 0.25,0.65,1.25$, and 1.65. These gaps originate from enhancement of localization due to long-range correlations with $\mu_{1}=0.2$ and $\mu_{2}=0.3$ [see Eq. (7)]. It is important that inside these gaps the transmission is practically zero, since the localization length is reduced by a factor of $W_{0} \approx 15.7$ and it becomes much less than the length of the waveguide.

Outside the gaps the transmission is $5 \%-10 \%$. This is a bit less than within a band for a periodic arrangement. At

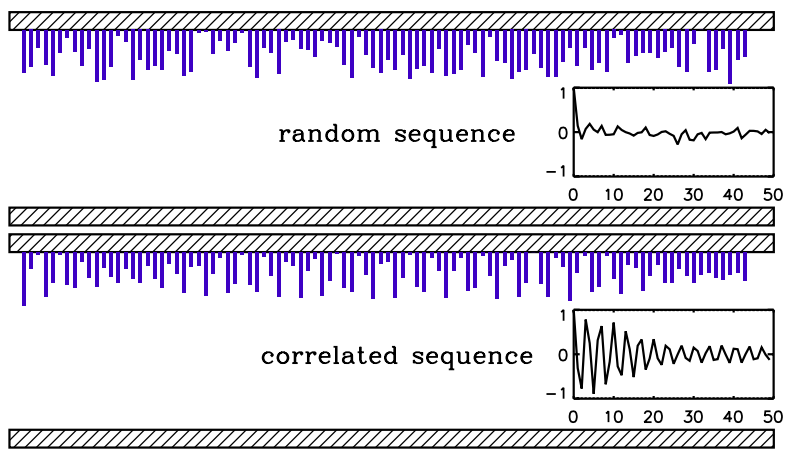

FIG. 2 (color online). Profile of intrusion of all 100 micrometer screws into the waveguide for uncorrelated and correlated random sequence $U_{n}$. The insets show the corresponding correlation function calculated from the micrometer screw depths [Eq. (7)]. 

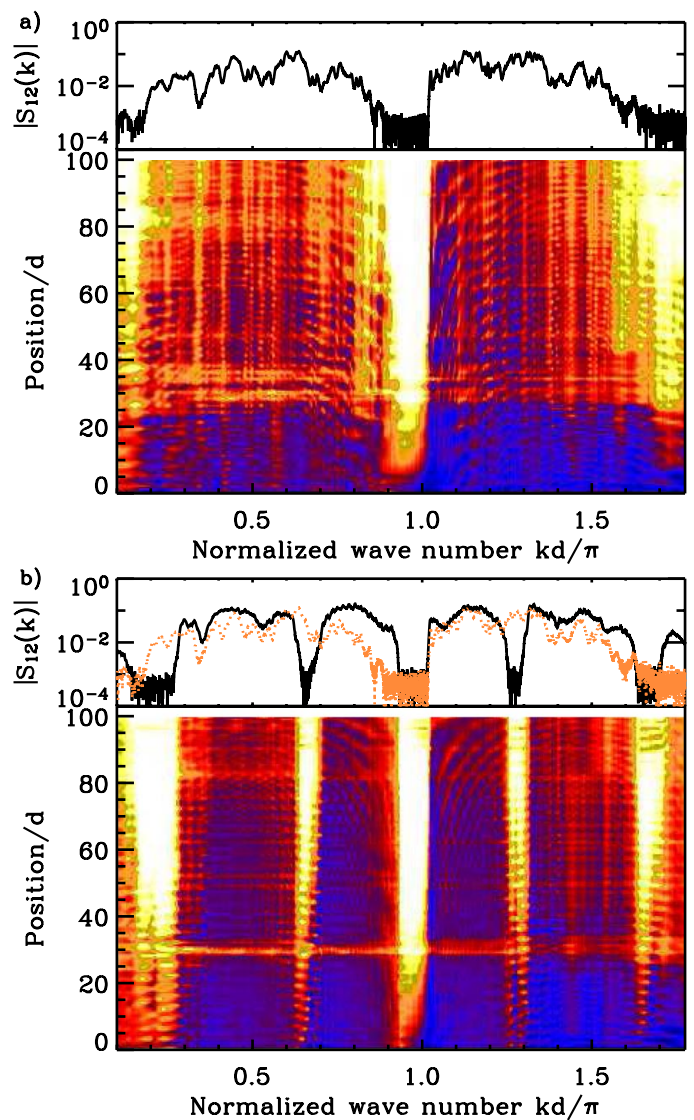

FIG. 3 (color online). Transmission spectrum $\left|S_{12}(k)\right|$ versus position $n$ (vertical scale) of the moving antenna is shown for uncorrelated (a) and correlated (b) disorder. The transmission spectrum $\left|S_{12}\right|$ through the whole waveguide is shown by black solid line at the top of each panel. For comparison, the transmission through uncorrelated disorder is added on the top of panel (b) by a yellow dotted line.

the same time it is larger for the correlated disorder case than in the uncorrelated one [see Fig. 3(b) top]. Since the relative size of the windows in which the transmission is practically zero is much less than the bandwidth, the integrated transmission for the correlated disorder is also larger than the one for the white-noise potential [see top of Fig. 3(b)]. The quantity that is conserved is the integrated logarithmic transmission. Because of the frequency dependence of absorption, and a noise level of $\left|S_{12}\right|^{2} \approx 10^{-6}$, it is not possible to obtain the integrated logarithmic transmission from the experiment.

The emergence of the additional gaps is also seen in Fig. 4, where the reflection pattern $\left|S_{22}\right|$ is shown for the correlated arrangement of scatterers. On top of the pattern, the reflection between the 41st and 42nd scatterer is shown. Although the localized states usually lead to strong decrease of $\left|S_{22}\right|$, we observe close to unity reflection within the gaps introduced by the correlations. Note that the fluctuations are strongly suppressed in comparison to the fluctuations in the band, e.g., at $k d / \pi=0.55$. This is even

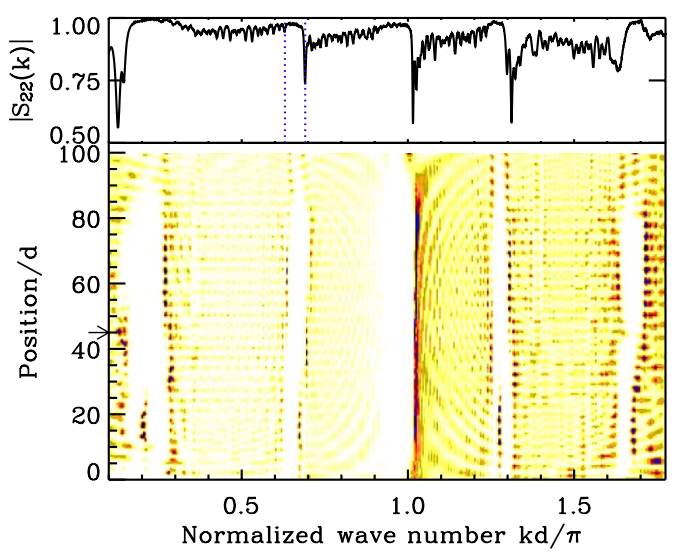

FIG. 4 (color online). The same as in Fig. 3(b) but for the reflection pattern $\left|S_{22}(k)\right|$. The value $\left|S_{22}\right|$ is shown for the antenna located between the 41 st and 42 nd scatterer.

better seen in Fig. 5 showing a narrow interval of the wave numbers close to the correlation gap at $k d / \pi \approx 0.65$. Several patterns with relatively high intensity of $\left|S_{22}\right|$ correspond to localized states, where the localization length is much smaller compared to the localization length in a white-noise arrangement. We denote these states as "enhanced-localized states." They belong to the spectrum of the system with long-range correlations Eq. (7). We observe that they are neither evanescent modes, nor defect states [19]. Both type of states may in principle appear within a "band gap" if some periodicity is introduced by the maxima of the correlation function. For evanescent modes the reflection $S_{22}$ would be close to 1, independently of the antenna position inside the sample. We stress that the local density of states does not change crucially by correlations. Since each pair of scatterers brings in one state, in the range $0.61 \leq k d / \pi \leq 0.71$ (shown in Fig. 5) there are about 10 states, assuming that the mean density of states is constant. Accordingly, we observe about 8 states in Fig. 5.

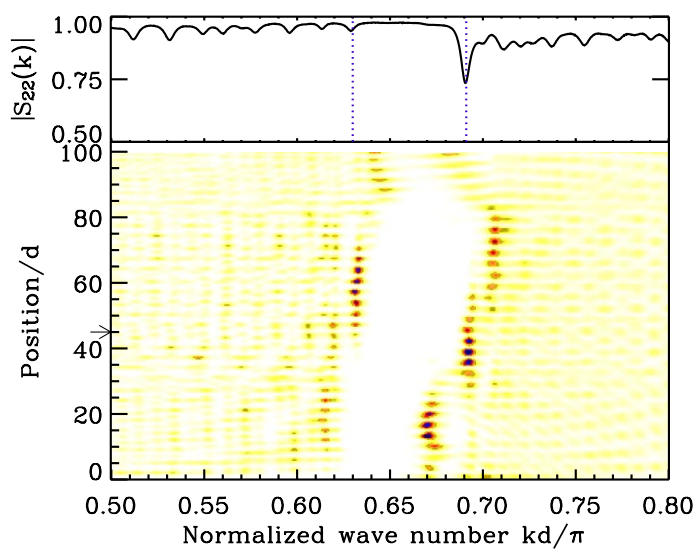

FIG. 5 (color online). Details of Fig. 4 for wave numbers between 0.50 and 0.80 . In this range of wave numbers one can see several enhanced-localized states. Sharp drop of $\left|S_{22}\right|$ at $k d / \pi \approx 0.691$ is due to localized states centered at $n \approx 40$. 


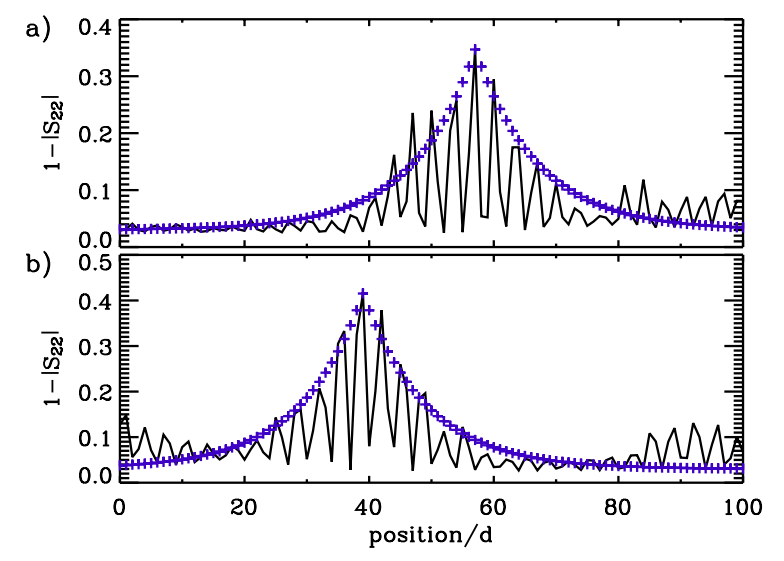

FIG. 6 (color online). Profiles of two enhanced-localized states at $k d / \pi \approx 0.63$ (a) and 0.69 (b) for the correlated sequence (marked by vertical dotted lines in Figs. 4 and 5. Graph (b) is the state responsible for a sharp decrease of $\left|S_{22}\right|$ shown at the top of Fig. 5. Crosses show an exponential decay with a localization length of 10 scatterer spacings.

Two enhanced-localized states are shown in Fig. 6. The quantity $1-\left|S_{22}\right|$, which is proportional to the intensity $|\psi|^{2}$ of the wave function [20], is plotted versus the coordinate of the moving antenna. Exponentially localized states are clearly seen inside the waveguide. The localization length is about 10 spacings $d$ between the scatterers. Thus, the emergence of enhanced-localized states due to long-range correlations is found experimentally. It is highly nontrivial that such strong enhancement of localization occurs for relatively weak fluctuations of the potential.

We would like to stress the good agreement between theory and experiment, in spite of the fact that (i) the analytical results are based on the analysis of the Lyapunov exponent for an infinite sample, (ii) the effect of long-range correlations is based on the binary correlator that is correct in the first Born approximation, (iii) the number of scatterers is quite small, and (iv) there is absorption of about $0.04 \mathrm{~dB}$ per unit cell $d$ for the empty waveguide. Also, the potential was scaled linearly to the micrometer screw depth, which is an approximation. However, a strong enhancement of localization due to long-range correlations is clearly seen, indicating that the observed effect is robust. The method may find various applications in the design of 1D structures especially as such localized states are controlled in the frequency space, a fact that may be important, for example, in random lasing $[15,16]$.

In conclusion, we performed experimental study of the effect of an enhancement of localization, that is due to specific long-range correlations in random potentials. Enhanced-localized states emerge inside the single-mode microwave waveguide, within two narrow frequency inter- vals. The enhancement factor (of about 16 in the experiment) for the localization length is inversely proportional to the width of frequency intervals. The positions of these intervals are in a good agreement with our theoretical predictions. These localized states appear for a quite small number of scatterers, $N=100$, thus the theory works well far beyond the region of its applicability.

This work was supported by the DFG via the Forschergruppe 760 "Scattering Systems with Complex Dynamics," and by the DOE Grant No. DE-FG0206ER46312.

[1] P. W. Anderson, Phys. Rev. 109, 1492 (1958).

[2] A. Z. Genack, Phys. Rev. Lett. 58, 2043 (1987).

[3] A. A. Chabanov, M. Stoytchev, and A.Z. Genack, Nature (London) 404, 850 (2000).

[4] T. Schwartz, G. Bartal, S. Fishman, and M. Segev, Nature (London) 446, 52 (2007).

[5] A.Z. Genack and A. A. Chabanov, J. Phys. A 38, 10465 (2005).

[6] I. M. Lifshitz, S. Gredeskul, and L. Pastur, Introduction to the Theory of Disordered Systems (Wiley, New York, 1988).

[7] N. M. Makarov, "Spectral and Transport Properties of One-Dimensional Disordered Conductors," http://www. ifuap.buap.mx/virtual/page_vir.html

[8] D. H. Dunlap, H.-L. Wu, and P. W. Phillips, Phys. Rev. Lett. 65, 88 (1990).

[9] P. Phillips and H.-L. Wu, Science 252, 1805 (1991).

[10] V. Bellani, E. Diez, R. Hey, L. Toni, L. Tarricone, G. B. Parravicini, F. Domínguez-Adame, and R. Gomez-Alcala, Phys. Rev. Lett. 82, 2159 (1999).

[11] F. A. B. F. de Moura and M. L. Lyra, Phys. Rev. Lett. 81, 3735 (1998).

[12] F. M. Izrailev and A. A. Krokhin, Phys. Rev. Lett. 82, 4062 (1999).

[13] U. Kuhl, F. M. Izrailev, A. A. Krokhin, and H.-J. Stöckmann, Appl. Phys. Lett. 77, 633 (2000).

[14] M. Titov and H. Schomerus, Phys. Rev. Lett. 95, 126602 (2005).

[15] H. Cao, Y. G. Zhao, S. T. Ho, E. W. Seelig, Q. H. Wang, and R. P. H. Chang, Phys. Rev. Lett. 82, 2278 (1999).

[16] M. Patra, Phys. Rev. E 67, 065603(R) (2003).

[17] U. Kuhl and H.-J. Stöckmann, Phys. Rev. Lett. 80, 3232 (1998).

[18] In fact, Eq. (6) is a conservation of the integrated Lyapunov exponent. This becomes clear if the function $W(2 \mu)$ is replaced [using Eq. (3)] by $l^{-1}(E) / l_{0}^{-1}(E)$, where $l_{0}(E)$ corresponds to white noise with $W(2 \mu)=1$.

[19] G. A. Luna-Acosta, H. Schanze, U. Kuhl, and H.-J. Stöckmann, "Transport Properties of One-Dimensional Photonic Crystals with Defects: Experiment and Theory" (to be published); arXiv:0711.1760.

[20] U. Kuhl, Eur. J. Phys. Special Topics 145, 103 (2007). 